than $1 \mathrm{ml}$ volume of saline solution leads to negative consequences for the patient.

\section{PO-0685 OUTCOME OF THYROID FUNCTION SCREENING IN BABIES BORN TO MOTHERS WITH AUTOIMMUNE HYPOTHYROID DISEASE}

A Gupta, S Balogan, S Sarangpani, C Harikumar. Paediatrics, University Hospital of North Tees, Stockton-on-Tees, UK

10.1136/archdischild-2014-307384.1324

Background and aims Autoimmune problems in mums are known to affect the newborn due to transplacental passage of antibodies. It Is reported that maternal autoimmune hypothyroidism can lead to transient but potentially serious effects in newborn babies warranting treatment. This has led to the practice of screening babies to help in early diagnosis and treatment. Unfortunately clinical practice in this area still suffers from conflicting evidence.

We carried out this retrospective review of our practice of assessing neonatal thyroid function in maternal autoimmune hyperthyroidism to help inform our practice locally whilst contributing to the discussion towards building a consensus nationally and internationally.

Methods Newborn babies born between January 2012 to March 2014 to mothers with autoimmune hypothyroid problems had their thyroid functions checked on day 3 and 10 respectively. They were also monitored clinically for signs and symptoms of hypo/ hyperthyroidism.

Results Overall 31 babies were screened in the study period, of which none warranted treatment for transient or permanent hypo/hyperthyroidism. The review also highlighted difficulties in implementing this guidance, as majority of the babies did not get investigated as per schedule.

Conclusion In our experience, babies born to mothers with auto-immune hypothyroidism did not develop transient hypothyroidism. There is a need for a larger scale study to look at the possible adverse effects of maternal autoimmune thyroid problems in the newborn.

\section{P0-0686 THE IMPACT OF VISCERAL OSTEOPATHIC TREATMENT ON THE MECONIUM EVACUATION IN VERY LOW BIRTH WEIGHT INFANTS}

N Haiden, A Kreissl, B Pimpel, A Berger. Pediatrics, Medical University of Vienna, Vienna, Austria

\subsection{6/archdischild-2014-307384.1325}

Objective To determine whether the complementary approach of manipulative osteopathic treatment accelerates complete meconium excretion and improves feeding tolerance in very low birth weight infants.

Methods This study was a prospective, randomised, controlled trial in premature infants with a birth weight $1500 \mathrm{~g}$ and a gestational age 32 weeks who received a visceral osteopathic treatment algorithm 3 times during their first week of life or no treatment.

Results Passage of last meconium occurred after a median of 7.5 days $(95 \%$ confidence interval: $6-9$ days, $\mathrm{n}=20$ ) in the intervention group and after 6 days (95\% confidence interval: 5-9 days, $\mathrm{n}=21)$ in the control group $(\mathrm{p}=0.11)$. However, osteopathic treatment was associated with a 12 day longer time to full enteral feedings $(\mathrm{p}=0.02)$, and a longer hospital stay (44 days longer in the intervention group; n.s). Osteopathic treatment was tolerated well and no adverse events were observed.

Conclusions Visceral osteopathic treatment oft the abdomen did not accelerate meconium excretion in VLBW-infants. However infants in the osteopathic group had a longer time to full enteral feedings and a longer hospital stay what must be interpreted as negative side effect. Further investigations are needed with modified protocols focussed on cranial osteopathy in this vulnerable group of patients. Currently the application of visceral osteopathic techniques cannot be recommended in VLBW-infants without further clinical trials.

\section{PO-0687 ALCOHOL USE IN PREGNANCY: GUIDELINES ON PRENATAL ARE SUFFICIENT?}

A Harff, S Nader, P Nader, S Fiamenghi. Neonatology, ULBRA/SSMD University Hospital, Canoas, Brazil

\subsection{6/archdischild-2014-307384.1326}

Background Alcohol use during pregnancy and the effects of exposure to the fetus have been described. The effects occur independent of the quantity consumed. Objective is identify alcohol use during pregnancy and check the orientation being given about the risk of alcohol use during prenatal care.

Design/methods 302 postpartum women were interviewed in the University Hospital ULBRA/Mãe de Deus from March to August 2012. We used the validated questionnaire T - ACE (Tolerance, Annoyed, Cut down, and Eye- opener) on the postnatal room pairs, featuring a random sample. The women were also asked about the performance of pre-natal and present guidelines on the use of alcohol.

Results Alcohol use during pregnancy was reported by 24\%. When used T - ACE was positive in $33.1 \%$ of subjects. During prenatal care, 219 women were instructed in prenatal and of these 72 (33\%) had positive T - ACE. Since the 68 women who were not oriented, 26 (35\%) are positive T-ACE group. No significance was found to have been targeted during the prenatal or not. Significant difference between the means of alcohol abuse $(\mathrm{T}-\mathrm{ACE})$ and the reported use during pregnancy $(\mathrm{p}<0.001)$, being higher in the instrument.

Conclusion It can be concluded that alcohol abuse found through validated instrument is high among pregnant women at the ULBRA/SSMD University Hospital. There was no difference in alcohol abuse among mothers counselled on the risk of alcohol use during pregnancy and not targeted during the prenatal period.

\section{PO-0688 ESTABLISHING PARENTHOOD IN THE BORDERLAND OF NORMALCY AFTER MODERATE OR LATE PRETERM BIRTH}

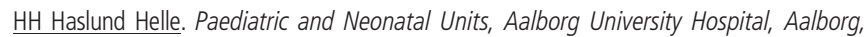
Denmark

\subsection{6/archdischild-2014-307384.1327}

Aim To investigate the process of establishing parenthood after birth of moderate or late preterm infant (GW 32-37).

Background Moderate or late preterm counts $80 \%$ of the premature population. In spite of known morbidity they are discharged without specialised health care services. Parents can 